\title{
Complicaciones Intrahospitalarias del Infarto de Miocardio Agudo Transmural en el Servicio de Cardiología del Hospital Nacional "Edgardo Rebagliati Martins". 1989-1993* \\ MARCOSPARIONA. GISLIAVORIVARA. RICARDOCASTII.LO

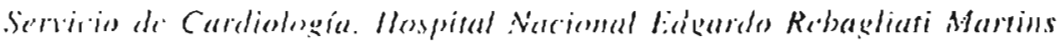

\begin{abstract}
RFSL WHE:
IE infarto de miocardio agndo constituye una de las principales causas de hospitalización y nuerte en el servicio de Cardiologia del llospital “Ed_aldo Rebagliati Martins». lil presente lrabajo pretende estudiar las características de las complicaciones del inlarto de miocarlio en nuestra población hospitalaria. para lo cual se revisó fos protocolos de 5.30 pacientes con diagnostico clinico. clectrocardiogrático y enzinatico de infario de miocardio acudo transmural hospitalizados en el servicio de Cardiologia del IINERII entre lo89 y 1993. Del total de

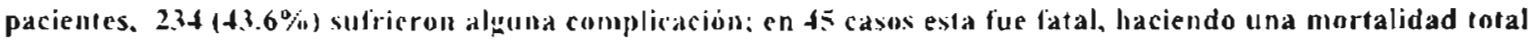
de $8.4 \%$ para nuesera scric. la insuliciencia ventricular izpuicrda fue la complicación más frecuente, presentánduse en 106 casos $(19,7 \%)$. llegando al shock cardiogcinico cn 28 pacientes (5.2\%). La taquicardia sinusual fue la arritmia nias frecuente. presentandose en 152 casos $(28.3 \%$ ). siguiéndole en orden de frecuencia la bradicardia sinusual con 88 casos ( $16,4 \%$ ). las estrasistoles ventriculares con 58 casos (10.8\%). la filorilación auricular con 40 casos $(7.5 \%$ ) y la tacuicardia ventricular y la tilsrilación ventricular con $8(1.5 \%)$ y 9 casos $(1.6 \%)$, respectivamente. (iénos que la inporlancia de este trabajo radica básicamente en la «cuantilicación» de las complicaciones! mortalidad por infarto de niocardio en nuestro medio. lo que redundará en una mejor usilización de los recursos parta el confrol de exta confermedad.
\end{abstract}

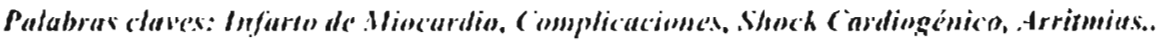

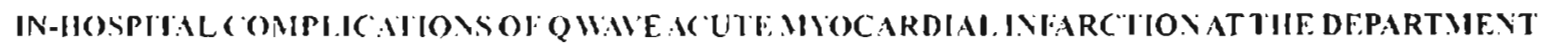

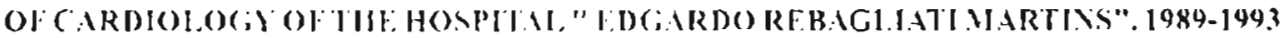

SLIMINRI:

Myocardial infarction is onc of the most inportant causes of admission and death at the Deparment of Cardiology

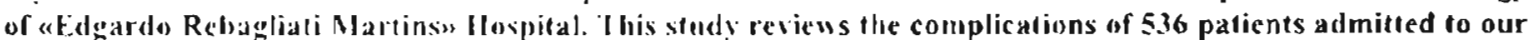
department with the diagnosis of $Q$ wale acutc myocardial infarction from 1989 to 1993. Out of these, 2.34 patients (4.3.6"\%) had a complication. in 45 cases it was fatal. and total mortality was $8.4 \%$. Pump failure was the most frequent complication, oceurred in 106 cases $(19.7 \%$ ). and turned to cardiogenic shock in 28 patients $(5,2 \%)$.

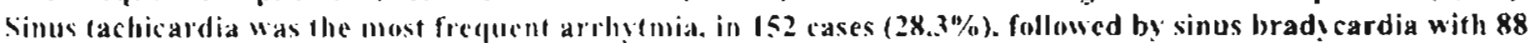
cases $(16.4 \%)$, premalure conericular contractions in 58 cases ( $10.8 \%)$. atrial fibrillation in 40 cases $(7.5 \%)$ and ventricular tach cardia and confricular fibrillation in $8(1.5 \%)$ and 9 cases $(1,6 \%)$ respectively. Is at this paper "quandifics" complications and nortalip from muscaldial infarction at our hospital in order 10 improve resources distribution in the control of this illness.

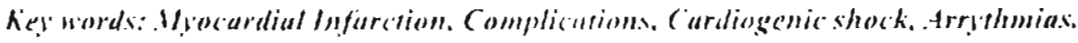

Correspondericia

Di: Rarcos Puliona

Aparada Posia! lik

Lima $l(t)$ - Pini

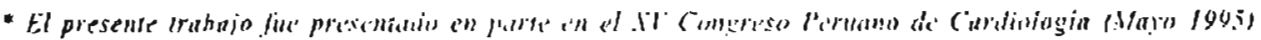




\section{INTRODUCCION}

El infarto de miocardio agudo constituye una de las causas más frecuentes de hospitalización en el servicio de Cardiología del Hospital «Edgardo Rebagliati Martins», asi como.una de las principales causas de muerte. En los Estados Unidos; casi 1.5 millones de personas sufren un infarto de miocardio agudo cada año, $y$ :cerca de la cuarta parte de todas las muertes se debe a un infarto de miocardio agudo (l').

Intervenciones tales como las unidades coronarias han contribuido a la reducción de la mortalidad. Sin embargo, la posibilidad de hacer más que observar pasivamente el curso de un infarto de miocardio agudo se ha convertido en una realidad sólo recientementé $\left({ }^{2-4}\right)$..

Con las mejoras en la monitorización electrocardiográfica y el tratamiento de las arritmias potencialmente letales que complican el infarto de miocardio agudo, la insuficiencia ventricular izquièrda y el shock cardiogénico representan la causa más frecuente de mortalidad entre los pacientes que ingresan en una unidad coronaria ( $\left.{ }^{5.6}\right)$.

La incidencia del shock tras el infarto de miocardio es del 10 al $15 \%$ y, con tratamiento conservador, dicha complicación se asöcia a una tasà de mortalidad del.80 al $100 \%\left({ }^{5,7}\right)$. Esta elevada tasa de mortalidad está principalmente relacionada con la extensa e irreversible pérdida de masa miocárdica ventricular izquierda $\left({ }^{8.50}\right)$. Los resultados de numerosos estudios clínicos (11,12) demostrando que las tasas de mortalidad intra-hospitalaria están drrectamente relacionadas a la fracción de eyección del ventriculo izquierdo han contribuido al concepto de que si hubiera reducción del tamaño del infarto, quizás, la tasa de mortalidad intra-hospitalaria disminuiria. Bengtson et al (1.3):encontraron, en una serie consecutiva de 200 pacientes con diagnóstico de infarto de miocardio agudo complicado con shock cardiogénico, que más de un tercio tenian un infarto de localización inferiet o posterior.

A pesar de la observación de los : serios efectos hemodinámicos del infarta ve ventriculo derecho casi dos décadas atrás, esta condicióntha rrecibido poca atención clínica hasta años recientes ( $\left.{ }^{14-16}\right)$ : Estudios post-mortem revelan que existe compromiso del ventrículo derecho en 19 a $51 \%$ de los pacientes cón infarto miocárdico agudo de localización inferior $(17.18)$. El infarto de ventrícula derecho contribuye marcadamente a intstabilidad hemodinámica, bloqueo de la conduccion aurículo-ventricular $y$ mortalidad intra-hospitalaria en pacientes con infarto de miocardio inferior ( ${ }^{16.19 .20}$ ). Un estudio realizado por Andersen et al.:-(2) sugiere que la elevación del segmento ST en las derivaciones V3R a'V6R es un marcador útil de $\mathrm{mal}$ pronóstico luego de un infarto de miocardio inferior.

Ciertas anomalías del ritmo cardiaco se observan en 72 a $96 \%$ de pacientes tratados en unidades coronarias. ' La frecuencia de arritmias es mayor en los pacientes que se observan tempranamente después del comienzol, de sintomas. Además muchas arritmias ocurrén antes de que el paciente llegue al hospital y, por lo tanto, sea monitorizado $\left({ }^{22}\right)$.
La bradicardia sinusal es la arritmia más frecuente que ocurre en fases precoces del infarto de miocardio agudo, especialmente en pacientes con infarto inferior y posterior $\left({ }^{23.24}\right)$.

A pesar de que el bloqueo caráiaco genteralmente responde a la terapia, ha sido asociado a un marcado incremento en la. mortalidad, la que parece estar relacionada a un mayor atriaño del infarto y a mayor compromiso de la función ventricular derecha e izquierda $\left({ }^{23-28}\right)$. Aún en los pacientes que reciben terapia trombolítica, el bloqueo auriculo-ventricular es común y está asociado a una mortalidad mayor $\left({ }^{29}\right)$.

Una frecuencia cardiaca elevada en los pacientes después de un infarto de miocardio agudo se considera generalmente un. marcador de función deprimida del ventrículo izquierdo y que refleja la severidad del daño miocárdico. Hjalmarson et al. $\left({ }^{30}\right)$ demostraron que tanto la mortalidad intra-hóspitalaria como la tatdia aumentaron con una frecuencia cardiaca incremeñtada.

La fibrilación auricular ocurre en cerca del $14 \%$ de los pacientes con infarto de miocardio agudo, usualmente asociada a insuficiencia ventricular izquierda, aunque la isquemia y la pericarditis pueden ser ocasionalmente la causa $\left({ }^{3}\right)$.

La fibrilación ventricular ocurre entre 1 a $11 \%$ de los pacientes con infarto de miocardio agudo. La fibrilación ventricular primaria $\left({ }^{32,13}\right)$ se define como la fibrilación veritricular que se presenta sin falla de bomba importante. Si ocurre tempranamente, el pronóstico es bueno entre los pacientes defibrilados exitosamente. Cuando la fibrilación ventricular es secundaria a falla de bomba, la mortalidad intrahospitalaria es mayor del $80 \%$.

La ruptura del septum interventricular ocurre en 0.5 a $1 \%$ de los casos de infarto de miocardio agudo, siendo el responsable del $5 \%$ de las muertes por esta patología. Se manifiesta con mayor frecuencia en los infartos de pared anterior, localizándose usualmente próxima al ápice del ventrículo izquierdo ( 34,15$)$. La evolución clínica depende de la severidad de la presentación inicial, pero en una forma general, $25 \%$ de los casos fallecen en shock cardiogénico en las primeras 24 horas, llegando al 85 de mortalidad en 2 meses con tratamiento médico solamente ( $\left.{ }^{36}\right)$.

El derrame pericárdico es frecuente y se observa en un $25 \%$ de los pacientes que han sufrido un infarto de miocardio agudo; se presenta especialmente en caso de infarto de localización. anterior, infarto de gran tamaño o insuficiencia cardiaca cangestiva $\left({ }^{37.38}\right)$.

La formación de trombos intraventriculares izquierdos es común en el desarrollo de un infarto de miocardio agudo, particularmente en el de localización anterior ( 39.40$)$.

El presente trabajo pretende estudiar las características del infarto de miocardio agudo en cuanto a sus complicaciones en ruestra población hospitalaria, como un aporte al conocimiento de la realidad hospitalaria y de salud en nuestro pais.

\section{MATERLAL Y METODOS}

Cop, el objeto de conocer las caracteristicas de las complicaciones del infarto miocárdico agudo transmural, se 
revisó los protocolos de infarto de miocardio agudo de 536 pacientes con diagnóstico clínico, electrocardiográfico, y enzimático de infarto de miocardio agudo transmural hospitalin zados en el servicio de Cardiologia del Hospital «Edgardo Rebagliati Martins», entre los años 1989 - 1993.

El protocolo de infarto de miocardio agudo incluye información clinica y de laboratorio sobre los antecedentes, la enfermedad actual, la evolución, el tratamiento y el pronóstico de los pacientes que son hospitalizados en nuestro servicio, por lo que puede ser considerado como una historia clínica breve. La elaboración de estos protocolos está encomendada a los médicos residentes de Cardiología y es revisada por el médico asistente encargado de realizar la epicrisis al alta hospitalaria.

Para verificar la significación estadística de las diferencias entre los grupos estudiados se utilizó la prueba del chi-cuadrado, considerándose un valor $p<0,05$ como estadisticamente significativo.

\section{RESULTADOS}

La edad promedio de los pacientes estudiados en esta revisión fue $64,9 \pm 11,8$ años, con un rango entre los 26 y 94 años $262(49 \%)$ de los pacientes tenían 65 o más años y 274 (51\%) eran menores de 65 años.

El sexo masculino fue el más afectado por la enfermedad, pues de los 536 pacientes, 428 (79,8\%) eran hombres y $108(20,2 \%)$ mujeres. Cabe acotar que entre los pacientes varones el infarto de miocardio agudo transmural fue más frecuente entre los menores de 65 años, $236(55,2 \%)$, que entre los mayores de 65 años, $192(44,8 \%)$. Lo opuesto ocurrió en el sexo femenino, donde el infarto de miocardio fue más frecuente entre las mayores de 65 años, $70(64,8 \%)$, que entre las menores de 65 años, $38(35,2 \%)$.

En lo que respecta a la localización del infarto, 307 casos $(57,28 \%)$ fueron de cara anterior y 229 casos $(42,72 \%)$ de cara inferior. Cuando se comparó por sexo, el infarto de cara anterior continuó predominando en los varones $(59,11 \%$ vs. $40,89 \%)$; no siendo así entre las mujeres, en las que la frecuencia fue similar para ambas localizaciones $(50 \%)$. En la comparación por grupo etáreo, el infarto de localización anterior es más frecuente tanto en los mayores de 65 años ( $58 \%$ vs. $42 \%$ ) como en los menores de 65 años $(56,5 \%$ vs. $43,5 \%)$.

\section{Complicaciones:}

Del total de 536 pacientes, $234(43,6 \%)$ sufrieron alguna complicación. En 45 casos las complicaciones fueron fatales, haciendo una mortalidad total del $8,4 \%$, para esta serie.

Cuando se comparó por grupo etáreo se encontró que entre los mayores de 65 años hubo 144 casos $(54,9 \%)$ que se complicaron, de los cuales $33(12,6 \%)$ fallecieron; y entre los menores de 65 años hubo 90 casos $(32,8 \%)$ complicados, de los cuales 12 $(4,4 \%)$ fallecieron, verificándose significación estadística tanto para las complicaciones $(p<0,01)$ como para la mortalidad $(p<0,01)$. Estas diferencias se mantuvieron cuando se desagregó por sexo, para cada caso $(p<0,01)$. Aunque se observó mayor frecuencia de complicaciones $(50 \%$ vs. $42 \%$ ) y mortalidad $(11,1 \%$ vs. $7,7 \%$ ) en el sexo femenino, esta diferencia no tuvo significación estadística $(p=0,8)$, aunque se mantuvo cuando se desagregó por edad (Tabla 1).

TABLA 1.- Infarto de miocardio agudo. Complicaciones y Mortalidad distribuidas por edad y sexo*

\section{GENERAL}

\begin{tabular}{lrrrrrr}
\hline & \multicolumn{2}{c}{ Total } & \multicolumn{2}{c}{$>65 \mathrm{a}$} & \multicolumn{2}{c}{$<65 \mathrm{a}$} \\
Total & 536 & $\%$ & 262 & $\%$ & 274 & $\%$ \\
\hline Complicaciones & 234 & 43,66 & 144 & 54,96 & 90 & 32,85 \\
Mortalidad & 45 & 8,40 & 33 & 12,60 & 12 & 4,38 \\
\hline
\end{tabular}

HOMBRES

\begin{tabular}{lrrrrrr}
\hline Total & 428 & $\%$ & 192 & $\%$ & 236 & $\%$ \\
\hline Complicaciones & 180 & 42,06 & 105 & 54,69 & 75 & 31,78 \\
Mortalidad & 33 & 7,71 & 23 & 11,98 & 10 & 4,24 \\
\hline
\end{tabular}

MUJERES

\begin{tabular}{lcccccr} 
Total & 108 & $\%$ & 70 & $\%$ & 38 & $\%$ \\
\hline Complicaciones & 52 & $\begin{array}{r}48,15 \\
\text { Mortalidad }\end{array}$ & 39 & 55,71 & 15 & 39,47 \\
\hline
\end{tabular}

*Valor de $p=<0,01$

En lo referente a las complicaciones por localización del infarto, se observó que el infarto de cara anterior tuvo mayor frecuencia de complicaciones $(48,5 \%)$ que el infarto de cara inferior $(37,1 \%)$, $(p<0,01)$. Así mismo, en cuanto a la mortalidad, el infarto de localización anterior tuvo una tasa mayor $(10,4 \%)$ que el de localización inferior $(5,6 \%),(p<0,05)$ (Tabla 2). Esta característica se mantuvo cuando se desagregó por sexo y grupo etáreo.

Con respecto al infarto de localización inferior, se observ6 una mayor frecuencia de complicaciones $(45,6 \%$ vs. $35,1 \%)$ y mortalidad $(9,0 \%$ vs. $4,8 \%)$ cuando había extensión a la cara posterior y/o lateral sin embargo, esta diferencia no alcanzó significación esładística $(\mathbf{p}<0,7)$ (Tabla 3 ).

\section{Insuficiencia ventricular izquierda - Shock cardiogénico:}

La insuficiencia ventricular izquierda se constituyó en la complicación intrahospitalaria más frecuente del infarto de miocardio agudo transmural en esta serie, presentándose en 106 casos (19,7\%) del total de infartos. El shock cardiogénico se presentó en 28 casos $(5,2 \%)$ del total de infartos. 
TABLA 2.- Infarto de miocardio agudo (IMA) : Complicaciones y Mortalidad distribuidos por localización del infarto, edad y sexo.

IMA ANTERIOR

TOTAL

HOMBRES

MUJERES

\begin{tabular}{rcccccccccccccccc} 
& TOTAL & COMPLIC. & $\%$ & MORTALI & $\%$ & TOTAL & COMPLIC. & $\%$ & MORTALI & $\%$ & TOTAL & COMPLIC. & $\%$ & MORTALI & $\%$ \\
\hline TOTAL & 307 & 149 & 48,53 & 32 & 10,42 & 253 & 117 & 46,25 & 23 & 9,09 & 54 & 32 & 59,26 & 9 & 16,67 \\
\hline$>65 a$ & 152 & 87 & 57,24 & 21 & 13,82 & 118 & 66 & 55,93 & 14 & 11,86 & 34 & 21 & 61,76 & 7 & 20,59 \\
$<65 \mathrm{a}$ & 155 & 62 & 40,00 & 11 & 7,10 & 135 & 51 & 37,78 & 9 & 6,67 & 20 & 11 & 55,00 & 2 & 10,00 \\
\hline
\end{tabular}

IMA ANTERIOR

\begin{tabular}{|c|c|c|c|c|c|c|c|c|c|c|c|c|c|c|c|}
\hline & & * & & ** & & & & & & & & & & & \\
\hline TOTAL & 229 & 85 & 37,12 & 13 & 5,68 & 175 & 63 & 36,00 & 10 & 5,71 & 54 & 22 & 40,74 & 3 & 5,56 \\
\hline$>65 a$ & 110 & 57 & 51,82 & 7 & 6,36 & 74 & 39 & 52,70 & 9 & 12,16 & 36 & 18 & 50,00 & 1 & 2,78 \\
\hline
\end{tabular}

$* p<0,001$

$$
\text { ** } \mathrm{p}<0,05
$$

En nuestra serie, 28 pacientes tuvieron una evolución fatal, haciendo una tasa de mortalidad del $5,2 \%$ para los pacjentes complicados con insuficiencia ventricular izquierda y/o shock cardiogénico, constituyendo esta complicación la principal causa de muerte intrahospitalaria por infarto de miocardio agudo transmural. Cuando se analizó por grupo etáreo, se observó que los mayores de 65 años fueron más afectados por esta complicacion $(26,3 \%$ hicieron insuficiencia ventricular izquierda y $7,2 \%$ shock cardiogénico), que los menores de 65 años (13,5\% hicieron insuficiencia ventricular izquierda y $3,2 \%$ shock cardiogénico), $(p<0,01)$. Asimismo, la mortalidad por esta causa es mayor entre los mayores de 65 años $(8,0 \%)$ que entre los menores de 65 años $(2,5 \%),(\mathrm{p}<0,01)$ (Tabla 4$)$.

Cuando se analizó por localización del infarto, se observó que los pacientes con infarto de localización anterior hicieron insuficiencia ventricular izquierda con más frecuencia que los afectados de infarto de localización inferior $(25,0 \%$ vs. $12,6 \%)$ $(p<0,01)$. Sin embargo, los pacientes con infarto de localización

TABLA 3.- IMA Inferior vs. IMA Inferior con extensión lateral y/o posterior. Complicaciones y mortalidad

\begin{tabular}{|c|c|c|c|c|c|}
\hline \multirow{2}{*}{ TOTAL } & \multicolumn{2}{|c|}{ IMA INFERIOR } & \multicolumn{2}{|c|}{$\begin{array}{l}\text { IMA INFERIOR } \\
\text { CON EXTENSION }\end{array}$} & \multirow[t]{2}{*}{$\begin{array}{l}\text { VALOR } \\
\text { DE } p\end{array}$} \\
\hline & 185 & $\%$ & 44 & $\%$ & \\
\hline Complicación & n 65 & 35,14 & 20 & 45,45 & $<0,7$ \\
\hline Mortalidad & 9 & 4,86 & 4 & 9,09 & N.S. \\
\hline
\end{tabular}

inferior que presentaron insuficiencia ventricular izquierda tuvieron una tasa de mortalidad mayor $(31,0 \%$ vs. $24,6 \%)$, no encontrándose significación estadística en esta diferencia $(p>0,9)$ (Tabla 5). Cabe señalar que, de los 106 pacientes que hicieron insuficiencia ventricular izquierda, 28 evolucionaron fatalmente, haciendo una tasa de mortalidad de $26,4 \%$ para esta complicación. De los 28 pacientes que hicieron shock, 25 fallecieron, haciendo una tasa de mortalidad del $89,2 \%$.

\section{Arritmias:}

La taquicardia sinusal fue la arritmia más frecuentemente encontrada, observándose en 152 casos $(28,3 \%)$; de este grupo, 13 pacientes evolucionaron fatalmente, haciendo una mortalidad relacionada de $8,5 \%$. La bradicardia sinusal fue la arritmia que siguió en orden de frecuencia, encontrảndose en 88 casos $(16,4 \%)$, de los cuales 5 fallecieron, obteniendose una mortalidad relacionada del $5,6 \%$.

Las extrasistoles ventriculares de todo tipo siguieron en

TABLA 4: Insuficiencia ventricular izquierda - Shock Cardiogénico. Distribución por edad

\begin{tabular}{|c|c|c|c|c|c|c|c|}
\hline \multirow{2}{*}{ TOTAL } & \multicolumn{2}{|c|}{ TOTAL } & \multicolumn{2}{|c|}{$>65 a$} & \multicolumn{2}{|c|}{$<65 a$} & \multirow{2}{*}{$\begin{array}{r}\text { VALOR } \\
\text { DE p }\end{array}$} \\
\hline & 536 & $\%$ & 262 & $\%$ & 274 & $\%$ & \\
\hline IVI & 106 & 19,78 & 69 & 26,34 & 37 & 13,50 & $<0,01$ \\
\hline $\begin{array}{l}\text { Mortalidad } \\
\text { Shock }\end{array}$ & $\begin{array}{l}28 \\
28\end{array}$ & $\begin{array}{l}5,22 \\
5,22\end{array}$ & $\begin{array}{l}21 \\
19\end{array}$ & $\begin{array}{l}8,02 \\
7,25\end{array}$ & $\begin{array}{l}7 \\
9\end{array}$ & $\begin{array}{l}2,55 \\
3,28\end{array}$ & $\begin{array}{l}<0,01 \\
<0,01\end{array}$ \\
\hline
\end{tabular}


TABLA 5: Insuficiencia ventricular izquierda - Shock Cardiogénico. Distribución por localización del infarto

\begin{tabular}{lccccc}
\hline \multirow{2}{*}{ TOTAL } & TOTAL & \multicolumn{2}{c}{ IVI } & Evolución al Shock \\
\cline { 2 - 6 } & 536 & 106 & $19,78 \%$ & 28 & $(26,42)$ \\
\hline & & $*$ & & & \\
IMA anterior & 307 & 77 & $25,08 \%$ & 16 & $(20,78)$ \\
IMA inferior & 229 & 29 & $12,66 \%$ & 12 & $(41,38)$ \\
\hline
\end{tabular}

( ) Porcentaje expresado tomando como total el número de pacientes con IVJ

$* \mathrm{P}<0,01$

orden de frecuencia, presentándose en 58 pacientes $(10,8 \%)$, de los cuales 7 fallecieron, haciendo una mortalidad asocjada de $12,0 \%$. La taquicardia ventricular se presentó en 8 casos $(1,5 \%)$, con una mortalidad relacionada de $25,0 \%$. La fibrilación ventricular se presentó en 9 casos $(1,6 \%)$, con una mortalidad relacionada de $55,5 \%$; se presentaron 3 casos de fibrilación ventricular primaria, sin mortalidad asociada.

La fibrilación aurícular ocupó el cuarto lugar en orden de frecuencia se presentó en 40 casos $(7,5 \%)$, con una mortalidad asociada de $5,0 \%$. Finalmente, la taquicardia supraventricular paroxística se presentó en 8 casos $(1,5 \%)$, sin mortalidad relacionada (Tabla 6).

Bloqueo de la conducción aurículo-ventricular o intraventricular:

Ef bloqueo AV de tercer grado fue el más frecuente, observándose en 24 pacientes $(4,5 \%)$, con una mortalidad asociada de $41,6 \%$. El bloqueo AV de segundo grado siguió en orden de frecuencia, encontrándose en 10 casos $(1,9 \%)$, con una mortalidad asociada de $30,0 \%$. El bloqueo AV de primer

TABLA 6 .- Arritmias: Frecuencias de presentación y mortalidad asociada.

\begin{tabular}{|c|c|c|c|c|}
\hline Arritmia & & cuencia & & $\begin{array}{c}\text { idad (\%) } \\
*\end{array}$ \\
\hline Extrasistoles ventric. & 58 & $10,82 \%$ & 7 & $12,07 \%$ \\
\hline Taquicardia ventric. & 8 & $1,49 \%$ & 2 & $25,00 \%$ \\
\hline Fibrilación ventric. & 9 & $1,68 \%$ & 5 & $55,56 \%$ \\
\hline Fibrilación auricular & 40 & $7,46 \%$ & 2 & $5,00 \%$ \\
\hline TSVP & 8 & $1,49 \%$ & 0 & $0,00 \%$ \\
\hline Taquicardia sinusal & 152 & $28,36 \%$ & 13 & $8,55 \%$ \\
\hline Extrasistoles ventric. & 88 & $16,42 \%$ & 5 & $5,68 \%$ \\
\hline
\end{tabular}

* Porcentaje del total de casos por arritmia grado se presentó en 4 pacientes $(0,7 \%)$, sin mortalidad relacionada (Tabla 7). Cuando se analizó por localización del infarto, el infarto de cara inferior se relacionó mas frecuentemente con los bloqueos AV de segundo $(90,0 \%)$ y tercer grado $(66,7 \%)$ (Tabla 8). En cuanto a los bloqueos de conducción intraventricular, la asociación de bloqueo de rama derecha + hemibloqueo anterior izquierdo ocurrió en 11 casos $(2,0 \%)$, con una mortalidad asociada de $27,2 \%$, constituyéndose asj́ en el tipo de bloqueo intraventricular más frecuente (Tabla 7).

\section{Infarto de ventrículo derecho:}

El diagnóstico de infarto de ventriculo derecho se realizó mediante dextrocateterismo con catéter balón guiado por flujo (Swan-Ganz) en todos los casos. Se verifícó 6 casos $(1,1 \%)$, de los cuales 4 fallecieron, haciendo una mortalidad relacionada de $66,6 \%$. En todos los casos se asoció a infarto de cara inferior.

TABLA ? - Bloqueos de la conducción aurículoventricular e intraventricular: Frecuencia de presentación y mortalidad asociada

\begin{tabular}{lrrrr}
\hline Bloqueo & \multicolumn{2}{c}{ Frecuencia } & \multicolumn{2}{c}{ Mortalidad (\%) } \\
\cline { 2 - 5 } BAV 3er grado & 24 & $4,48 \%$ & 10 & $41,67 \%$ \\
BAV 2do grado & 10 & $1,87 \%$ & 3 & $30,00 \%$ \\
BAV ler grado & 4 & $0,75 \%$ & 0 & $0,00 \%$ \\
BRD + HBAI & 111 & $20,71 \%$ & 3 & $2,70 \%$ \\
BRD + HBPI & 0 & $0,00 \%$ & 0 & $0,00 \%$ \\
BRD & 10 & $1,87 \%$ & 0 & $0,00 \%$ \\
BRI & 2 & $0,37 \%$ & 2 & $100,00 \%$ \\
HBAI & 7 & $1,31 \%$ & 0 & $0,00 \%$ \\
HBPI & 0 & $0,00 \%$ & 0 & $0,00 \%$ \\
\hline
\end{tabular}

* Porcentaje del total de casos por bloqueo

BRD: Bloqueo de rama derecha

BRI: Bloqueo de rama izquierda

HBAI: Hemibloqueo anterior izquierdo

HBPI: Hemibloqueo posterior izquierdo

TABLA 8. - Bloqueo de la conducción auriculo-ventricular. Distribución por localización de infarto

\begin{tabular}{lccccccc}
\hline & & BAV ler Grado & \multicolumn{2}{c}{ BAV 2do Grado } & \multicolumn{2}{c}{ BAV 3er Grado } \\
\cline { 2 - 8 } TOTAL & 4 & $\%$ & 10 & $\%$ & 24 & $\%$ \\
\hline IMA anterior & 2 & 50,00 & 1 & 10,00 & 8 & 33,33 \\
IMA inferior & 2 & 50,00 & 9 & 90,00 & 16 & 66,67
\end{tabular}




\section{Pericarditis:}

Se encontró 15 casos $(2,8 \%)$, de los cuales uno fallcció, haciendo una mortalidad asociada de $6.6 \%$. Su hallazgo fue más frecuente en los infartos de cara anterior (10 casos) que en los de cara inferior ( 5 casos) (Tabla 9).

\section{Complicaciones mecánicas:}

La ruptura del septum interventricular fue observada en 7 casos $(1,3 \%)$, de los cuales 6 fallecieron (mortalidad: $85,7 \%$ ); se asoció a infarto de cara anterior en 5 casos y a infarto de cara inferior en 2 casos.

Se encontró un caso de insuficiencia mitral aguda, el cual se asoció a infarto de cara anterior, con evolución fatal.

\section{Miscelánea:}

Se halló crisis hipertensiva en 12 casos $(2.2 \%)$.

Se registró 15 casos $(2,8 \%)$ de accidente cerebro-vascular, de los cuales 13 se asociaron a infarto de cara anterior y 2 a infarto de cara inferior.

Se obscrvó un caso de embolia pulmonar, asociado a infarto de cara inferior.

\section{DISCUSION}

En nuestra serie de 536 pacientes, el infario de miocardio agudo transmural fue largamente más frecuente entre los varones, quienes sufrieron casi el $80 \%$ del total de infartos, comprobándose, como es ya bien sabido, que el sexo masculino es un factor de riesgo importante de cnfermedad coronaria. Tambićn se comprueba en nuestra serie que en el sexo femenino el infarto de miocardio es más frecuente entre las mujeres postmenopáusicas $\left({ }^{+1}\right)$, quienes fueron afectadas en el $65 \%$ del total de infartos sufridos por el sexo femenino.

La mortalidad intrahospitalaria total para la población estudiada fue $8,4 \%$, discretamente por debajo del promedio norteamericano ('). La edad fue un factor predictivo significativo de complicaciones y mortalidad, siendo la mortalidad intrahospitalaria para los mayores de 65 años de $12.6 \%$, tasa similar a la encontrada por Weaver y col $\left({ }^{52}\right)$.

En lo que respecta a la localización del infarto. el infarto de cara anterior tuvo una tasa mayor de complicaciones y mortalidad que el infarto de cara inferior, siendo eslas diferencias significativas estadisticamente $(p<0,01$ y $p<0,05$. respectivamente), hallazgo que concuerda con el de orros estudios $\left({ }^{3+4}\right)$.
Se ha demosirado que cuanto mayor es la extensión del infarto, peor es el pronóstico del paciente $\left(^{8 \cdot 12}\right.$ ). Sin embargo, en nuestra serie. cuando comparamos los infartos de cara inferior con y sin extensión postcrior y/o lateral, a pesar de haber mayor número de complicaciones $y$ muertes en el primer grupo, estas diferencias no fueron estadisticamente significativas $(p<0,7)$.

\section{Insuficiencia ventricular izquierda - Shock cardiogénico:}

La insuficiencia ventricular izquierda y el shock cardiogénico fueron la causa principal de muerte en el grupo estudiado, dato que concucrda con lo observado a nivel mundial ("5). El shock cardiogénico se presentó en $5.2 \%$ de los casos: lo cual está por debajo del promedio, aunque la mortalidad por esta complicación $(89.2 \%)$ sí concuerda con lo referido por la literatura $\left({ }^{\circ}\right)$.

La edad fue un factor predictivo tanto para la presentación de shock cardiogénico como para un peor pronóstico, concordando con lo descrito en pacientes estudiados en otras series $(5.7 .13)$.

Los pacientes con infarto de localización anterior hicieron insuficiencia ventricular izquierda con mayor frecuencia que los afectados por un infarto de cara inferior, siendo esta diferencia estadisticamente significativa. Sin embargo, si consideramos sólo a los pacientes que evolucionaron a shock cardiogénico, el $42 \%$ de estos eran pacientes con infarto de cara inferior. Este hallazgo contrasta con los resultados de otra serie ( ), en que el $80 \%$ de los pacientes tenian un infarto de cara anterior, pero es similar a los de otras series $\left(^{5.13}\right)$.

\section{Arritmias:}

Debido a características propias de nuestro hospital, la mayoria de los pacientes con infarto de miocardio agudo son hospitalizados entre las $24-48$ horas del inicio de los sintomas, por lo que probablemente gran número de arritmias, que ocurren en la primeras 24 horas $\left({ }^{22}\right)$, no hayan sido registradas en los protocolos de infarto que sirvieron de información para este trabajo.

La arritmia mas frecuentemente encontrada fue la taquicardia sinusal, a diferencia de otras series $\left({ }^{23.2 \mathrm{~J}}\right)$, en las que este lugar le correspondia a la bradicardia sinusal, que en nuestra revisión ocupó el segundo puesto en orden de frecuencia. Aunque una frecuencia cardiaca aumentada se asocia a aumento de la mortalidad intrahospitalaria, en nuestra población la taquicardia sinusal tuvo una mortalidad asociada de $8,5 \%$, la cual es mucho menor a la referida en otra serie $\left({ }^{30}\right)$.

TABLA 9.- Infarto de miocardio agudo: Otras complicaciones. Distribución por localización del infarto

\begin{tabular}{|c|c|c|c|c|c|c|c|c|c|c|}
\hline \multirow[b]{2}{*}{ TOTAL } & \multicolumn{2}{|c|}{ Pericarditis } & \multicolumn{2}{|c|}{ Ruptura SIV } & \multicolumn{2}{|c|}{ Insuf. Mitral Aguda } & \multicolumn{2}{|c|}{ Crisis HTA } & \multicolumn{2}{|c|}{$A C V$} \\
\hline & 15 & $\%$ & 7 & $\%$ & 1 & $\%$ & 12 & $\%$ & 15 & $\%$ \\
\hline IMA anterior & 10 & 66,67 & 5 & 71.43 & 1 & 100,00 & 10 & 83,33 & 13 & 86,67 \\
\hline IMA inferior & 5 & 33,33 & 2 & 28,57 & 0 & 0,00 & 2 & 13,33 & 13 & 86,67 \\
\hline
\end{tabular}


La frecuencia de arritmias ventriculares en nuestra serie es mucho menor que la referida por la literatura, esto probablemente debido a la relativa demora en la hospitalización de nuestros pacientes. Cabe acotar que la fibrilación ventricular fue la artitmia más letal, con $55,5 \%$ de mortalidad asociada. Sin embargo, tal como se ha referido anteriormente $\left({ }^{32} .33\right)$, cuando se trata de la forma primaria (sin falla de bomba asociada) la mortalidad es baja; en nuestra serie se presentaron 3 casos, todos fueron defibrilados exitosamente y evolucionaron favorablemente.

\section{Bloqueo de la conducción aurículo-ventricular o intraventricular:}

El bloqueo aurículo-ventricular de grado alto (segundo y tercer grado) se presentó en $6.4 \%$ de todos los infartos y en $10,9 \%$ de los infartos de cara inferior, frecuencia más baja que la reportada por otras series $\left({ }^{16.29}\right)$. Su presencia se asoció a una mortalidad alta, 30,0\% para el bloqueo AV de segundo grado y $41,6 \%$ para el bloqueo AV de tercer grado. La presencia de bloqueo de rama izquierda fue altamente letal $(100 \%)$ en nuestra serie; no ocurrió asi con el bloqueo de rama derecha que no se asocio a mortalidad, hallazgo discordante con lo referido en otros estudios $\left({ }^{45}\right)$.

\section{Infarto de ventrículo derecho:}

En nuestra serie, la presentación de esta complicación es infrecuente, pues no alcanza ni al $3 \%$ de los infartos de cara inferior, lo que difiere grandemente de otros estudios, en los cuales alcanza hasta el $50 \%$ de los infartos inferiores $\left({ }^{17 .}{ }^{18}\right.$ ). Probablemente esto se deba a la poca atención clinica que recibe esta complicación $\left({ }^{14.16}\right)$ y a la ausencia de un criterio diagnóstico exacto (15.16.30). Su presencia se asocia a mortalidad incrementada ( $66,6 \%$ en nuestra serie), lo que coincide con otras revisiones $\left({ }^{16.19}\right)$. Probablemente, el uso rutinario de derivaciones derechas en los infartos inferiores sea útil $\left({ }^{(3)}\right)$ en el diagnóstico, cuando menos presuntivo, de esta complicación.

\section{Complicaciones mecánicas:}

La ruptura del septum interventricular se produjo en el 1,3\% de los pacientes con infarto de miocardio agudo en nuestra revisión. Se presentó más frecuentemente en los infartos de localización anterior y tuvo una mortalidad del $85 \%$. Todos estos hallazgos concuerdan con lo encontrado en otras series $\left({ }^{34-36}\right)$.

\section{Accidente cerebro-vascular:}

En la población estudiada, se presentaron 15 accidentes cerebro-vasculares, que representan el $2,8 \%$ del total de infartos registrados. Se asociaron a infarto de cara anterior en $13(86,6 \%)$ de los casos, lo cual podria estar en relación a la relativamente frecuencia alta con que se forman trombos intraventriculares izquierdos, especialmente en los infartos de localización anterior $\left({ }^{39} \cdot 10\right)$.

\section{CONCLUSIONES}

El presente trabajo es, a nuestro conocimiento, la serie más larga que examina las complicaciones intrahospitalarias del infarto de miocardio agudo transmural en nuestro medio y confirma en la mayoria de los casos los datos obtenidos en otros medios, en lo referente a variables demográficas, tales como edad y sexo, el pronóstico de acuerdo a la localización del infarto, y la frecuencia de las complicaciones (insuficiencia ventricular izquierda/shock cardiogénico, arritmias y transtornos de la conducción, etc.). Asi mismo, nos brinda datos numéricos sobre las complicaciones y monalidad en el infarto de miocardio agudo que no distan de los promedios informados en medios de alra sofisticacion. Cabe acotar que, debido a las caracierísticas de nuestro hospital el grupo estudiado constituye en cierta forma un grupo «seleccionado», debido a que su ingreso al servicio de Cardiología no se produce siempre en las primeras horas de inicio de síntomas y esto produce un sesgo en la información obtenida, lo cual garantiza un nuevo estudio que involucre a todos los pacientes con infarto de miocardio agudo desde su ingreso al hospital.

Finalmente, creemos que la importancia del presente estudio radica básicamente en la "cuantificación» de las complicaciones durante el infarto de miocardio agudo en nuestro medio, lo cual permitirá un mejor manejo de estrategias y recursos para el control de esta enfermedad.

\section{BIBLIOGRAFIA}

1) American Heart Association: Hearl facls. Dallas, Texas. American Heart Association National Center, 1987.

2) Ellis SG. Interventions in acule myocardial infarction. Circulation 81 (Suppl IV):43, 1990.

3) Goldman L, Cook EF. The decline in ischemic heart disesse mortality rates. An analysis of the comparative effects of medical inlerventions and changes in lifestyle. Ann Intern Med 101:825, 1984.

4) Beaglebole R. Medical management and the decline in mortality from coronary heart disease. Br Med J 292:33, 1986.

5) Scheidt S, Ascheim R, Killip T. Shock after acute myocardial infarction: A clinical and hemodynamic profile. Am J Cardiol 26:556. 1970.

6) Ong L, Green S, Reiser P, Morrison J. Early prediction of mortatity in patients with acute myocardial infarction: A prospective study of clinical and radionuclide risk faciors. Am J Cardiol 57:33, 1986.

7) Honds ME, Rutherford JD, Muller JE et al. The in-hospital development of cardiogenic shock after myocardial infarction: Incidence, predictors of ocurrence, outcome and prognostic factors. J Am Coll Cardiol 14:40, 1989.

8) Page DL, Caulfield JB, Kastor JA, De Sanctis RW, Sanders CA. Myocardial changes associated with cardiogenic shock. N Eng J Med 285:133, 1971.

9) Gutovitz AL, Sobel BE, Roberis R. Progressive nature of myocardial injury in selected patients with cardiogenic shock. Am J Cardiol 41:469, 1978. 
10) Geddes JS, Adgey AAJ, Pantridge JF: Prevention of cardiogenic shock. Am Hearl J 99:243, 1980.

11) Kelly MJ, Thompson PL, Quinlan MF. Prognostic significance of left ventricular ejection fraction after acute myocardial infarction: A bedside radionuclide study. Br Heart J 53:16, 1985.

12) Shah PK, Pichler M, Berman DS, Singh B., Swan HJ, Left ventricular ejection fraction determined by radionuclide ventriculography in early stages of first transmural myocardial intarclion: Relation to short-term prognosis. Am J Cardiol 45:542, 1980.

13) Bengston JR, Kaplan AJ, Pieper KS, Willerman N.M, Mark DB, Pryor DB, Phillips HR, Califf RM. Cardiogenic shock in evolving myocardial infarction: Prognosis in the interventional era. J Am Coll Cardiol 20:1482, 1992.

14) Cobn JN, Guiba NH, Broder Mll, Limas CJ: Right ventricular infarction: Clinical and hemodynamic features. Am J Cardiol 33:209, 1974.

15) Cohn JN. Right ventricular infarclion revisited. Am J Cardiol 43:666. 1979.

16) Berger PB, Ryan TJ. Inferior myocardial infarction: High risk subgroups. Circulacion 81:401, 1990.

17) Forman MB, Goodin J, Phelan B, Kopelman H, Virmani R. Electrocardiographic changes associated with isolated right ventricular infarcrion. J Am Cols Cardiol 4:640, 1984

18) Isner J.M, Roberts WC. Right ventricular infarction complicating left ventricular infarction secondary to coronary artery discase: Frequency, location. associated findings and significance from analysis of 236 necropsy patients with acute or healed myocardial infarction. Am J Cardiol 42:885, 1978.

19) Shab PK, Maddahi J, Berman DS, Pichler M, Swan HJC. Scintigraphically detected predominant right ventricular dysfunction in acute myocardial infarction: Clinical and hemodynamic correlates and implications for theraphy and prognosis. I Am Coll Cardiol 6:1264, 1985

20) Rodrigues EA, Dewhurst NG, Smart LM, Hannab WJ, Muir AL. Diagnosis and prognosis of right ventricular infarction. $\mathrm{Br}$ Heart J 56:J9, 1986.

21) Andersen HR, Nielsen D, Lund O, Falk E. Prognostic significance of right ventricular infarction diagnosed by ST elevation in right chest leads V3R to V7R. Int J Cardiol 23:349, 1989.

22) O'Doherty M, Tayler D1, Quion E, Vincent R, Chamberlain DA. Five hundred patients with myocardial infarction monitored within one hour of symploms. Br Med J 286:1405, 1983.

23) Adgers AAJ, Alley JD, Geddes JS, James RGG, Webb SW, Zaidi SA.Acule phase of myocardial infarction. Lancel 2:501, 1971.

24) Groner LE, Gershen BJ, Orlando NM, Epstein SE. Bradycardia and its complications in the pre-hospital phase of acute myocardia! infarction. Am J Cardiol 32:607, 1973.

25) Tans AC, Lie KI, Durrer D. Clinical setting and prognostic significance of high degree atrioventricular block in acute myocardial infarction: A study of 144 patients. Am Heart J 99:4, 1980.

26) Nicod P, Gilpin E, Dittrich H, Polikar R, Henning H, Ross J. Long-term outcome in patients with inferior myocardial infarction and complete atrioventricular block. J Am Coll Cardiol 12:589, 1988.

27) Sclarovsky S, Strasberg B, Hirshbey A, Arditi A, Lewin RF, Agmon J. Advanced early and late atrioventricular block in acute inferior wall myocardial infarction. Am Heart J 54:985, 1984.

28) Feigl D, Astkenazy J, Kíshon Y. Early and late atrioventricular block in acute inferior myocardial infarction. J Am Coll Cardiol 4:35, 1984.
29) Berger PB, Ruocco NA, and the TIMI Investigators. Incidence and prognostic implications of heart block complicating inferior myocardial infarction treated with thrombolytic theraphy: Results from TIMI II. J Am Coll Cardiol 20:533, 1992.

30) Hjalmarson A, Gilpin EA, Kjekshus J, Schieman G, Nicod P, Henning $\mathbf{H}$, Ross J. Influence of heart rate on mortality after acule myocardial infarction. Am J Cardiol 65:547. 1990.

31) Hed H, Lew HS, Kettai M, Corcek B, Geft IO,Shah P, Ganz W. Early atrial fibrillation during evolving myocardial infarction: A consequence of impaired left atrial perfusion. Circulation 75:146, 1987.

32) Volpi A, Maggioni A, Franzosi MG, Pampallona S, Mauri F, Tognoni G. In-hospital prognosis of patients with actite myocardial infarction complicated by primary ventricular fibrillation. $\mathrm{N}$ Eng $\mathrm{J}$ Med 317:257, 1987

33) Ruskin JN. Primary ventricular fibrillation. N Eng J Med 317:307, 1987.

34) Hutchios JM. Rupture of the interventricular septum complicating myocardial infarction: Pathological analysis of 10 patients with clinical diagnosed perforation. Am Heart J 97:165, 1979.

3 S) Viodaver E, Edwards JE. Rupture of ventricular septm or papillary muscle complicating myocardial infarcion. Circulation 55:815, 1977.

36) Fendey MP, Chang VP, O'Rourke MF. Myocardial rupture after acule myocardial infarciion: Ten year review. Br Heart J 49:550, 1983.

37) Pinard LA, Albert A, Henrard L, Lempereur P, Sprynger $M$, Carlier J, Kulberteu HE. Incidence and significance of pericardial effussion in acute myocardial infarction as determined by two-dimensional echocardiography. J Am Coll Cardiol 8:517, 1986.

38 ) Wunderink RG. Incidence of pericardial effusion in acute myocardial infarctions. Chest 85:492, 1985.

39) Ezekowitz MD. Acute infarction, left ventricular thrombus and systemic embolization: An approach to management. J Am Coll Cardiol 5:1281, 1985

40) Nordrehaug JE, Jobannessen KA, Von der Lippe G. Usefulness of high dose anticoagulants in preventing left ventricular thrombus in acute myocardial infarction. Am J Cardiol 55:1941, 1985.

4 1) Stampfer MJ, Willett WC, Colditz GA, Rosner B, Speizer FE, Hennekens $\mathrm{CH}$. A prospective study of posimenopausal estrogen therapy and coronary heart disease. N Eng J Med 313:1044, 1985.

42) Weaver WD, Litwin PE, Martin JS, Kudeachuk PJ, Maynard C, Eisenberg MS, Ho MT, Cobb LA, Kennedy JW, Wirkus MS, MITI Project Group. Effecl of age on use of thrombolytic theraphy and mortality in acule myocardial infarction. I Am Coll Cardiol 18:657, 1991.

43) Gruppo Italiano per lo Studio della Streptochinasi nell' Infarto Miocardico (GISSI). Effectiveness of intravenous thrombolytic treatment in acute myocardial infarction. Lancet 1:397, 1986.

44) ISIS-2 (Second International Study of Infarct Survival) Collaborative Group. Randomized trial of intravenous streptokinase, oral aspirin, both, or neither among 17,817 cases of suspected acule myocardial infarction. ISIS-2. Lancet 2:349, 1988.

45) Hindman MC, Waguer GS, JaRo M, Atkins JM. The clinical significance of bundle branch block complicating acute myocardial infarction: Clinical characteristics, hospital mortality, and one-year follow-up. Circulation 58:679, 1978. 